\title{
Total internal reflection imaging ellipsometry (TIRIE) biosensor sensitivity improvement with low noise imaging device
}

\author{
Li Liu ${ }^{a}$, Gang Jin a, \\ ${ }^{a}$ NML, Institution of Mechanics, Chinese Academy of Sciences, \#15, Bei-si-huan West \\ Road, Beijing 100190, China; \\ * Corresponding author. Tel./fax: +86 10 82544138. E-mail address: gajin@imech.ac.cn.
}

\begin{abstract}
The biosensors with properties of real-time, high throughput and label-free are more and more popular recently, in which the biosensor based on the total internal reflection imaging ellipsometry (TIRIE) is a novel imaging detector for protein interaction processes. In previous work, three techniques are introduced to improve the performance of the biosensor including polarization setting optimization, spectroscopic light source application and low noise CCD detector adoption. In this paper, the effect of the low noise CCD detector technology on the sensitivity and detection limit improvement is analyzed. An obvious improvement of 10 time increase in the sensitivity and SNR, and 50 times lower concentration in the detection limit is obtained by optimization.
\end{abstract}

Keywords: total internal reflection imaging ellipsometry, biosensor.

\section{Introduction}

Imaging biosensor is an effective tool for the visualization and quantitative measurement of the bio-molecule interaction [1]. Imaging ellipsometry biosensor has developed more than 15 years since its concept proposed [2] and nowadays it is a powerful tool used in bio-molecule detection. Ellipsometry is a phase-sensitive method and it has very high sensitivity which reaches to the order of sub-angstrom of a thin film at the interface (As a phase-sensitive method, it enjoys very high sensitivity, in order of sub-angstrom against changes of average thickness of films at the interface.) [3]. Imaging ellipsometry achieves the high sensitivity and high-throughput ability in bio-medical and clinical detection [4]. Several years ago, an imaging ellipsometry immuno-sensor and micro-fluidic system was developed for detecting protein concentration in solution [5,6]. Furthermore, total internal reflection imaging ellipsometry (TIRIE) was developed, which is a micro-fluidic reactor array combined with imaging ellipsometry for bio-molecular interaction real-time detection. As described in Ref. [7], TIRIE biosensor is based on the phase sensitive ellipsometric property and the large view field property of the imaging ellipsometry. In addition, the micro-fluidic reactor array system is applied for biomolecule interactions and sampling, to provide the high sensitivity and high throughput property.

Recent years, researchers are focusing on the performance improvement of the biosensor such as sensitivity, the SNR (signal and noise ratio), the detection limit etc. to solve various detection in bio-medical field. In order to improve the performance of the TIRIE biosensor, three optimization procedures are brought in, including spectroscopic light source application, polarization optimization and low noise imaging adoption, the content is introduced elsewhere. In this paper, the effect of the low noise CCD detector to the performance of the biosensor is analyzed. A comparison between the imaging system without and with low noise function is shown. Finally, HbsAg and its antibody interaction process are shown in optimized condition to display the sensitivity and the detection limit improvement of the biosensor.

Sensors, Cameras, and Systems for Industrial, Scientific, and Consumer Applications XII,

edited by Ralf Widenhorn, Valérie Nguyen, Proc. of SPIE-IS\&T Electronic Imaging, SPIE Vol. 7875

$78750 U$ · (C) 2011 SPIE-IS\&T · CCC code: 0277-786X/11/\$18 - doi: 10.1117/12.871978 


\section{TIRIE biosensor Setup}

Figure 1 is the schematic diagram of the TIRIE biosensor. The TIRIE biosensor combines the optical system with a micro-fluidic protein reactor array system. The optical system is consisted of an imaging ellipsometry with a light source of Xe lamp and a wavelength selector to form an expanded collimating light probe of single wavelength. The light beam from the optical fiber goes through the polarizer, the compensator (a quarter wave plate) and penetrates into the coupling prism perpendicularly. The light beam is reflected totally at the interface of the substrate and passes through an analyzer, and penetrated to the imaging system which is an imaging lens and the imaging device of CCD. The azimuth setting of the polarizer, the compensator and the analyzer is adjusted in a range from $0^{\circ}$ to $180^{\circ}$.

The sampling of the biosensor is used the micro-fluidic protein reactor array system. It is made of poly(dimethylsiloxane) (PDMS). The micro-array reactor is attached to a gold layer surface on glass to form 24 individual cell arrays. Each cell has an inlet and an outlet for the solution. Any kinds of molecule solution can be individually delivered to each cell by the micro-fluidic system. By the micro-fluidic reactor array system, the sample consumption of the biomolecule solution reaches about $15 \mu \mathrm{l}$ and the test time normally is effectively minimized to tens of minutes. The optimized flux is $5 \mu \mathrm{l} / \mathrm{min}$. Moreover, a high throughput (24 channels) detection can be easily achieved with the combined TIRIE with a micro-fluidic reactor array system.

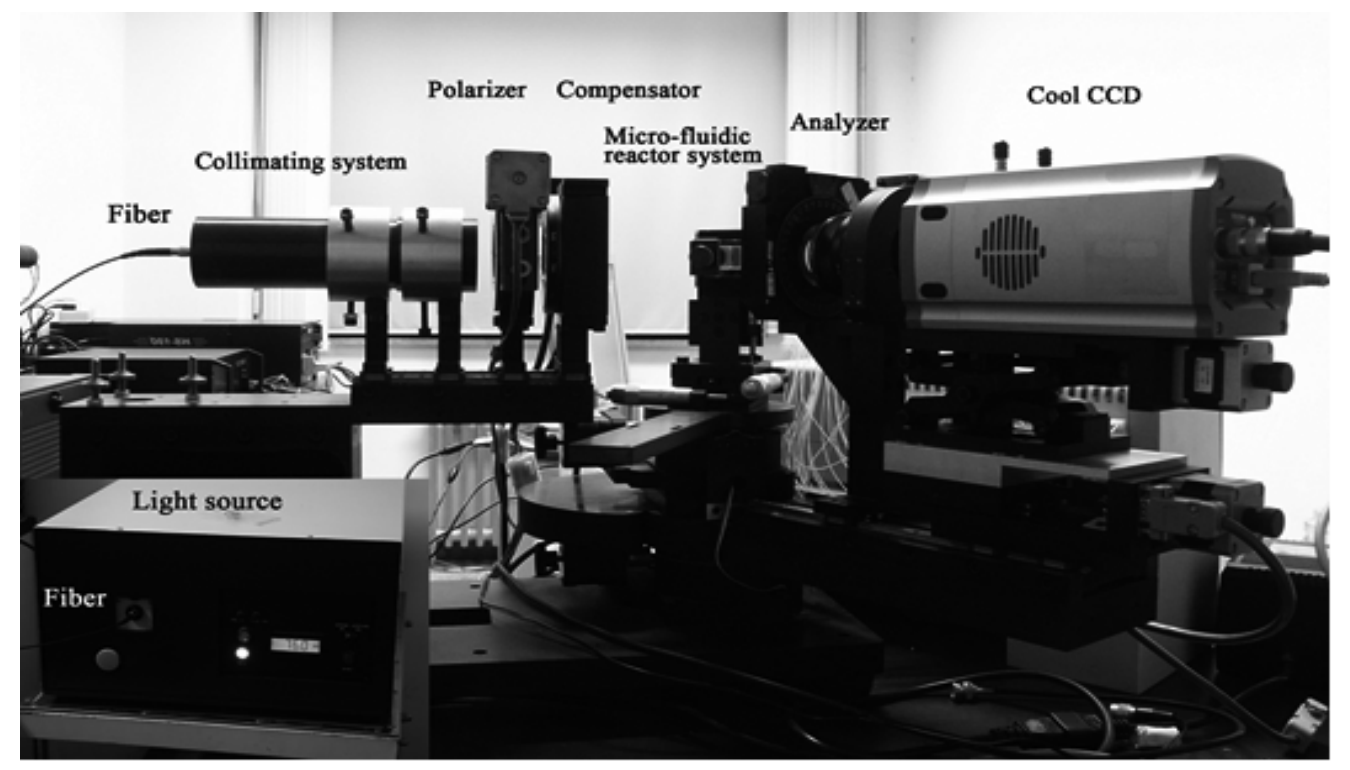

Figure 1. Schematic diagram of the total internal reflection imaging ellipsometry biosensor.

\section{Imaging system improvement}

Generally, the sensitivity and the detection limit are restricted by the noise of the device, so that the performance can be improved by decreasing the noise or increasing the signal to noise ratio. In the TIRIE biosensor, the noise comes from temperature fluctuation in imaging device, light source noise, as well as from the flow disturbance in micro-fluidic reactor. A general approach to reduce the thermally induced noise is to implement a temperature controller [1] in the imaging device, that is, an imaging CCD with low noise. High imaging quality such as high spatial and time resolution can be obtained by imaging CCD with low noise. In order to illustrate the effect of the imaging CCD with low noise, two imaging system are applied to detect the protein layer. The parameters of the system are shown below.

In the previous TIRIE biosensor system, the imaging device is a video CCD (Sony Japan) without the temperature control. The camera features a corresponding spectrum range of $400-900 \mathrm{~nm}$, sensitive area of $768 \times 576$ pixels and $6.0 \times 4.96 \mathrm{~mm}^{2}$ (single pixel size of $6.5 \times 6.25 \mu^{2}$ ). The electronic gain is fixed for quantitative photonic intensity measurement. The sampling speed is 30 frame/S at maximum. The 
nonlinearity of the CCD camera is better than $1 \%$. The image signal is inputted into an image grab board (Matrox, Canada) inserted in the computer for analog-to-digital (A/D) conversion and the digital image processing and storage. The ellipsometric images are recorded in 8-bit (0-255) grayscale format [2]. However, the sensitivity and the detection limit of the biosensor are not quite satisfactory and can hardly satisfy the demands of biological detection.

In the new system, a scientific low noise imaging CCD camera (Andor DU937N-BV,) with low noise function is used as the imaging device of the TIRIE biosensor. The camera features a corresponding spectrum range of $300-1000 \mathrm{~nm}$, sensitive area of $512 \times 512$ pixels and $6.6 \times 6.6 \mathrm{~mm}^{2}$ (single pixel size of $13 \times 13 \mu \mathrm{m}^{2}$ ). The electronic gain is $x 1, x 2$ and $x 4$ for chosen. The ellipsometic images are recorded in 16-bit (0-65535) grayscale format. The read noise $10.3 \mathrm{e} @ 2.5 \mathrm{MHz}$, dark current $0.0005 \mathrm{e}-/ \mathrm{pixel} / \mathrm{sec}$ and the low imaging CCD with temperature control from 0 to -100 degree.

With low read noise and a temperature control, the thermal noise is reduced effectively by the cool CCD. Meanwhile the size of each pixel of the cool CCD is 2 times bigger than that of the video CCD. The saturation intensity is increased and the dynamic range of imaging is enlarged as well. The sample will be detected in a broad intensity range.

In order to illustrate the function of the cool CCD in the biosensor measurement, a $3.5 \mathrm{~nm}$ thick IgG adsorption protein layer is imaged by cameras with low noise CCD and the video CCD. The exposure time of both cameras is $0.004 \mathrm{~s}$ and 100 serial pictures are captured. The even grayscale of 100 pixels of one picture is taken as signal $S_{n}(n=1,2, \ldots \ldots 100)$. The experiment results are normalized by the even value of 100 signals, as shown in Fig.2. Here, the mean square root is taken to measure the noise of the biosensor. The noise of the biosensor without and with cool CCD is $5 \times 10^{-3}$ and $4.5 \times 10^{-4}$, respectively. The noise is decreased by almost one order of magnitude. It is concluded that the cool CCD with low noise as the imaging device can improve SNR by more than 10 times.

In practice, a cool CCD is much more expensive than a normal one, so it is only used for high sensitivity demands. The temperature of the CCD during the detection is $-70^{\circ}$. The pre-gain of the CCD is 2 and the dynamic range in grayscale is from 0 to 65535 .

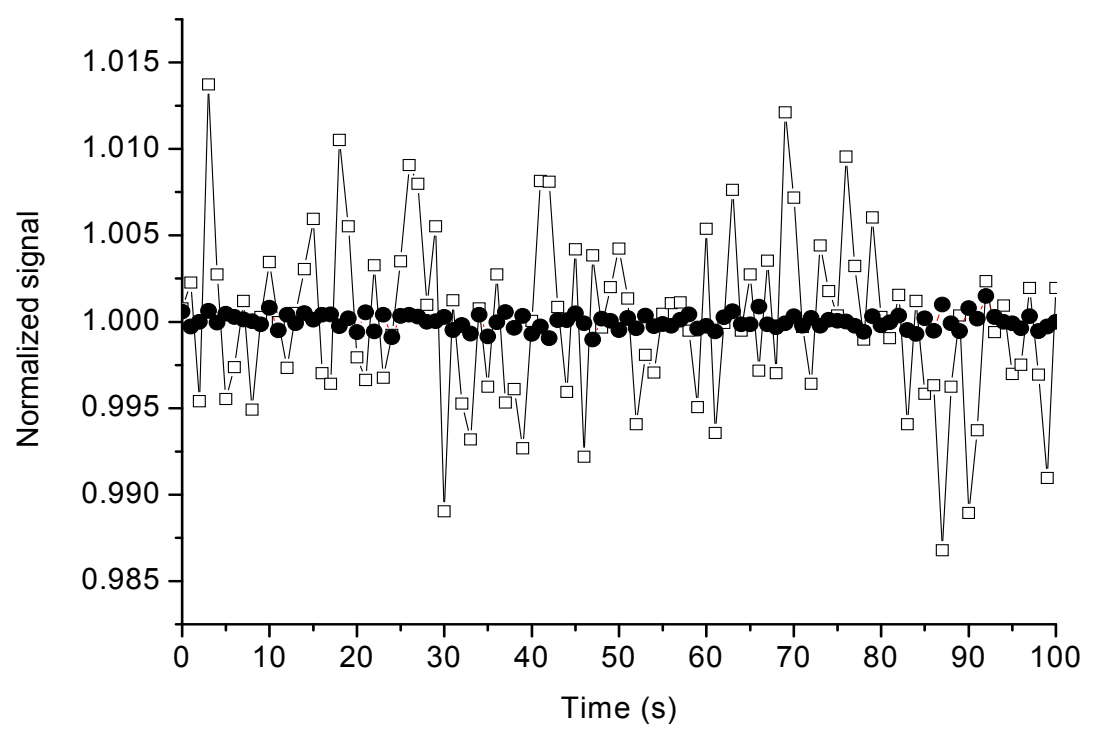

Figure 2 The normalized signals of the $3.5 \mathrm{~nm}$ thick IgG protein layer obtained by the biosensor with video CCD (“”) and cool CCD (“•"). 


\section{Experiments}

An experiment is performed to verify the performance improvement of the biosensor. The quantitative detection of Hepatitis B surface antigen ( $\mathrm{HBsAg}$ ) is concerned. The optical conditions of the biosensor are as described above.

\subsection{Optical system}

The incident angle is $59^{\circ}$. According to the work previously[10], the wavelength is $600 \mathrm{~nm}$ and the azimuth setting of polarizer and analyzer is $122^{\circ}$ and $44^{\circ}$, respectively. The cool CCD is used as an imaging device. The temperature is set at $-70^{\circ}$, pre-gain is $\mathrm{x} 2$.

\subsection{Materials}

The SF10 glass slides were purchased from Changchun Institute of Optics, Fine Mechanics and Physics, Chinese Academy of Sciences (China). The 11-mercaptoundecanoic acid (MUA) from Sigma (USA), 1-(3Dimethylaminopropyl)-3-ethylcarbodimide hydrochloride (EDC) and N-hydroxy-droxysuccinimide (NHS) from ACROS, and all chemicals for preparing phosphate-buffered Saline (PBS, $10 \mathrm{mM}$ phosphate, $0.1 \mathrm{M} \mathrm{NaCl}, \mathrm{pH}$ 7.4) from Sigma also. De-ionized water (with resistivity of $18.3 \mathrm{M} \Omega \mathrm{cm}$ ) was produced by ion exchange demineralization, followed by passing through a Milli-Q plus system from Millipore (Millipore, Bedford, MA). Hepatitis B virus surface antibody and surface antigen were provided by Hotgen company (Beijing China) and Blocking buffer from Sigma (USA).

\subsection{Substrate}

The SF10 glass slide was prepared by the evaporation of $2 \mathrm{~nm}$ chromium on the surface and $30 \mathrm{~nm}$ gold layer was used as the substrate. The gold surface was immersed into MUA- ethanol solution for at least 18 hours, followed by a thorough rinsing with both ethanol and de-ionized water. The MUA monolayer with carboxyl group was self-assembled on the gold surface.

\section{Results}

The process of HbsAb binds with HbsAg is shown in Fig.3. The $1^{\text {st }}$ region is the baseline. The $2^{\text {nd }}$ region is surface activation with NHS and EDC; $3^{\text {rd }}$ is rinsing with PBS buffer. $4^{\text {th }}$ is the Hepatitis B surface antibody (HBsAb) which is used as the ligand covalently immobilized on the gold surface with carboxyl group, for 10 min. $5^{\text {th }}$ region is surface rinsing with PBS buffer for about $6 \mathrm{~min} .6^{\text {th }}$ is surface blocking with blocking buffer for $10 \mathrm{~min} ., 7^{\text {th }}$ is surface rinsing with PBS buffer for $6 \mathrm{~min} .8^{\text {th }}$ region is HbsAg interacting with ligand. The HBsAg is diluted with PBS buffer into various concentrations from 0 to $250 \mathrm{ng} / \mathrm{ml}$ and incubated with ligand for interactions upon their affinity for $15 \mathrm{~min}$. The signal is taken in an average result over each sensing area in each cell in the array of 100 pixels. The curves in Figure 3 are zoomed into "region 7" and "region 8" to show the dynamic process of the interaction in detail on the surface. Curves from "a" to " $g$ " correspond to $\mathrm{HbsAg}$ concentrations of $0,8,16,32,63,125,250 \mathrm{ng} / \mathrm{ml}$. The data in "region 8 " is averaged over the last $600 \mathrm{~s}$, as indicated in the Figure 3. 


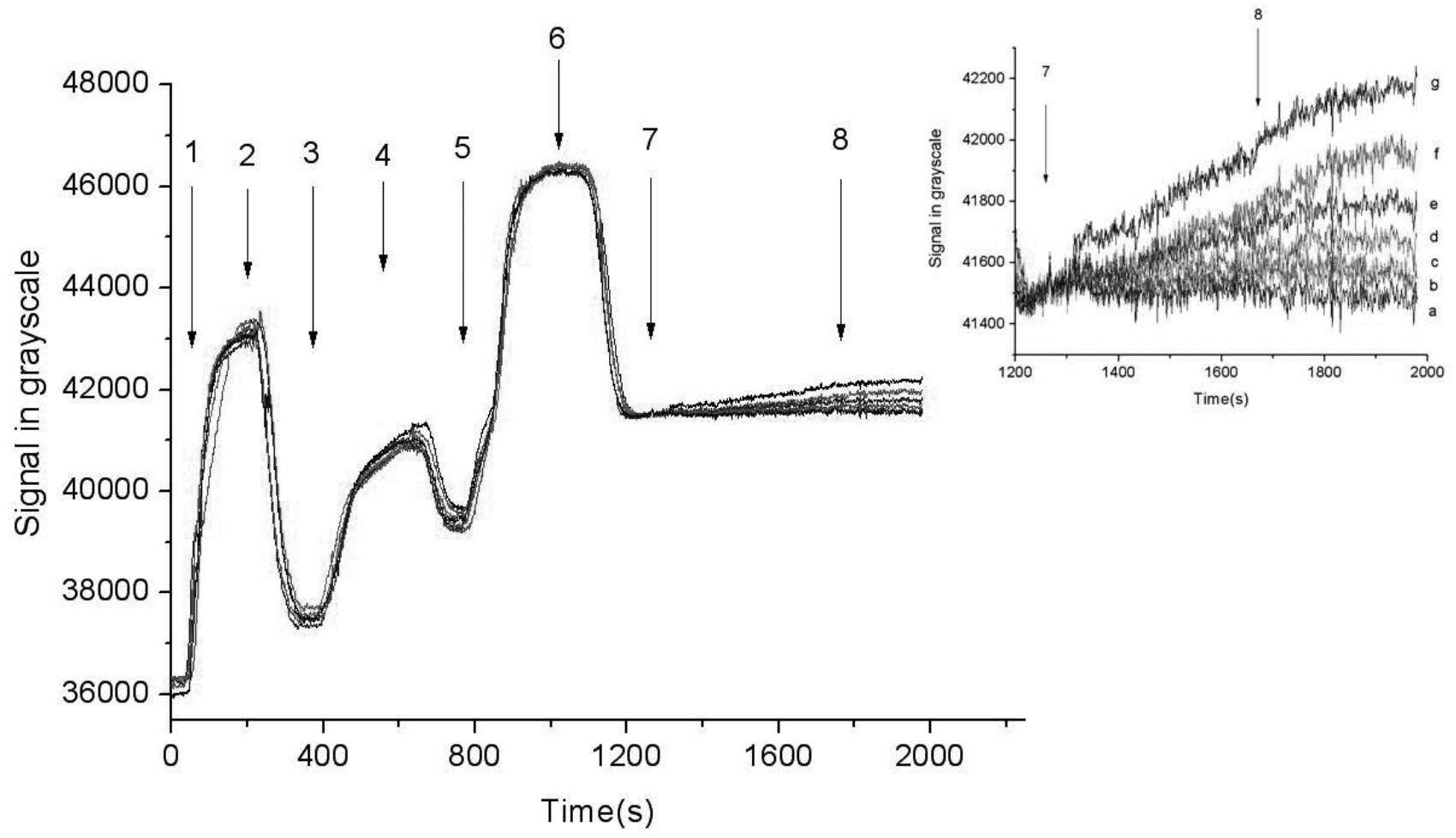

Figure 3 Real-time curves of the dynamic process for the Hepatitis B virus surface antigen binding with Hepatitis $B$ surface antibody in various concentrations. The $1^{\text {st }}$ region is the baseline; the $2^{\text {nd }}$ region NHS-EDC activation of carboxyl group assembled on the gold substrate; $3^{\text {rd }}-$ PBS rising; $4^{\text {th }}-\mathrm{HBsAb}$ immobilization on the gold-coated substrate surface; $5^{\text {th }}-$ PBS rinsing, $6^{\text {th }}-$ blocking, $7^{\text {th }}-$ PBS rinsing and $8^{\text {th }}-\mathrm{HBsAg}$ binding with its antibody. Curves a-h are for Hepatitis $B$ virus surface antigen concentrations of $0,8,16,32,63,125,250 \mathrm{ng} / \mathrm{ml}$.

\section{Discussions}

Figure 4 shows kinetic curves of HBsAb reacting with various concentrations of HBsAg for the previous system and the improved system. The transduction signals are normalized by the mean value of the initial 100 s of the dynamic curves, as the baseline of the interaction. Curves " $D$ " to " $A$ " with concentrations 400, $1600,3200,6400 \mathrm{ng} / \mathrm{mL}$ are obtained by the previous biosensor system. The wavelength is set as $633 \mathrm{~nm}$ and the imaging device is the video CCD camera (Sony XC-ST30 CCD B/W video camera). Curves "k" to "e" with concentrations of $0,8,16,32,63,125,250 \mathrm{ng} / \mathrm{ml}$ are obtained by the improved biosensor with optimized conditions mentioned above. 


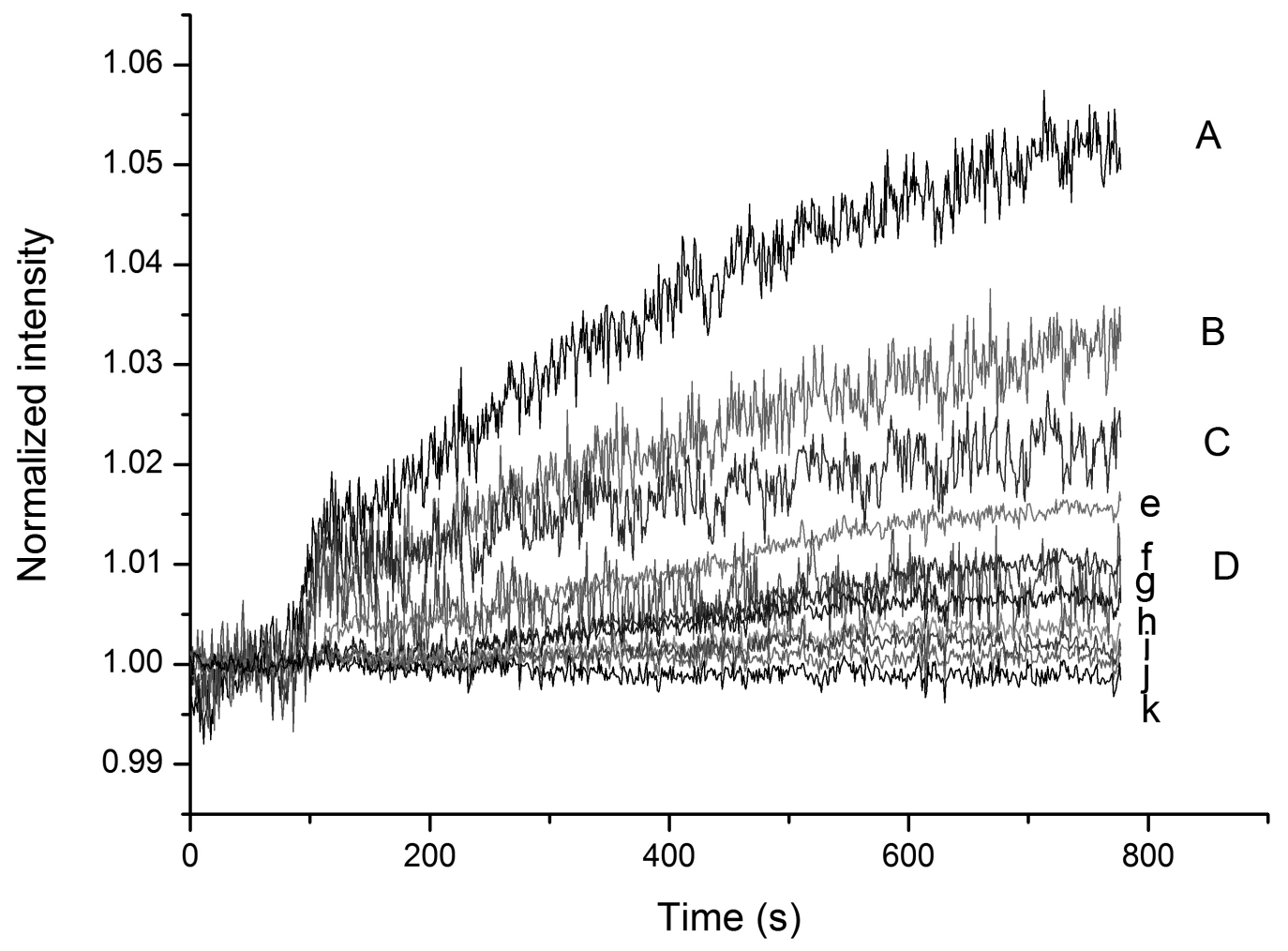

Figure 4 Dynamic curves of $\mathrm{HbsAg}$ reacting with $\mathrm{HbsAb}$ : curves $\mathrm{D}$ to $\mathrm{A}$ represent the normalized signal with concentrations of 400 to $6400 \mathrm{ng} / \mathrm{ml}$ measured without optimized conditions; curves " $\mathrm{k}$ " to "e" represent the normalized signal with concentrations of 0 to $250 \mathrm{ng} / \mathrm{ml}$ measured under optimized conditions.

The detection limit of the previous biosensor is $400 \mathrm{ng} / \mathrm{mL}$, as being affected by the noise. The signal can hardly be distinguished when the concentration is lower. The performance of detection limit is improved from $400 \mathrm{ng} / \mathrm{mL}$ to $8 \mathrm{ng} / \mathrm{mL}$ by optimizing the setting of the wavelength, the azimuth setting of the polarizer and the analyzer as well as by using the cool CCD. The detection limit in the improved biosensor system is enhanced by about 50 times. SNR is increased obviously, so that the lower concentrations from $0-250 \mathrm{ng} / \mathrm{ml}$ could be distinguished, which is impossible in the preliminary system. With optimized conditions, the sensitivity is improved effectively, which results in the normalized transduction response to $250 \mathrm{ng} / \mathrm{ml}$ even higher than $400 \mathrm{ng} / \mathrm{ml}$ in preliminary one.

\section{Conclusions}

In this paper, the effect of the low noise imaging system is shown. The SNR improves almost 1 order by the cool CCD. The detection limit of the biosensor is improved almost 50 times in HbsAg detection.

In other hand, the TIRIE biosensor, especially with improved performance will be a powerful tool for protein analysis with properties of label-free, multi-target parallel detected, high sensitivity, low consumption of reagents and real-time analysis for protein interaction process. The potential application in detection of biomolecule weak-affinity interaction and clinic test with high throughput is foreseen now. 


\section{ACKNOWLEDGEMENT}

The author gratefully acknowledges the support of National Basic Research Program of China 2009CB320300, National High Technology Research and Development Program (863) of China (2008AA02Z419), Chinese Academy of Sciences (KJCX2-YW-M03 and -M04), and National Natural Science Foundation of China 20805053.

\section{REFERENCES}

[1] Jin, G., Tengvall, P., Lundstrom, I., and Arwin, H., "A Bionsensor concept based on imaging ellipsometry for visualization of biomolecular interactions," Anal. Biochem. 232(1), 69-72 (1995)..

[2] Jin, G., Jansson, R., and Arwin, H., "Imaging ellipsometry revisited: Developments for visualization of thin transparent layers on silicon substrates," Rev. Sci. Instrum. 67(8), 2930-2936 (1996).

[3] R.M.A.Azzam, N.M.Bashara, Ellipsometry and Polarized Light, North-Holland, (1977).

[4] P. Van Gerwen, W. Laureyn, A. Campitelli, P. Jacobs, P. Detemple, K. Baert, W. Sansen, R. Mertens, Journal of Micromechanics and Microengineering 10/3 (2000).

[5] Wang, Z. H. and Jin, G., "A label-free multisensing immunosensor based on imaging ellipsometry," Anal. Chem. 75(22), 6119-6123 (2003).

[6] Jin, G., "Development of biosensor based on imaging ellipsometry," Phys. Status Solidi A 205(4), 810-816 (2008).

[7] Y.Y. Chen, J. Gang, The Ninth World Congress on Biosensors.Canada: Biosensors \& Bioelectronics, 219 (2006).

[8] X.D. Fan, I.M. White, S.I. Shopoua, H.Y. Zhu, J.D. Suter, Y.Z. Sun, Analytica Chimica Acta 620/1-2 (2008) 8.

[9] Meng, Y. H. and Jin, G., "Technique of grabbing and processing in ellipsometric imaging system," Opt. Precis. Eng. 8 316-320 (2000).

[10] Liu, L., Chen, Y. Y., Meng, Y. H., Chen, S., and Jin, G., "Improvement for sensitivity of biosensor with total internal reflection imaging ellipsometry (TIRIE)," Thin Solid Films In press (2011). 